\title{
Scattering of light by a periodic structure in the presence of randomness IV. Limit of detection by curve fitting
}

\author{
$\mathrm{S} \mathrm{CHATTERJEE}^{1}$ and V C VANI ${ }^{2}$ \\ ${ }^{1}$ Indian Institute of Astrophysics, Bangalore 560 034, India \\ ${ }^{2}$ Department of Instrumentation, Indian Institute of Science, Bangalore 560 012, India \\ E-mail: chat@iiap.ernet.in; chat@bgl.vsnl.net.in; vani@isu.iisc.ernet.in
}

MS received 22 November 2004; revised 18 April 2005; accepted 23 May 2005

\begin{abstract}
In the context of scattering of light, we determine the extent of randomness within which a hidden periodic part can still be detected. The detection is carried out using a technique called the extended matched filtering, first introduced by us in this context. The earlier prediction, before our technique was introduced, had placed the limit of detection, by intensity measurements alone, at $\left(r_{0} / \Lambda\right) \sim 0.33$, where $r_{0}$ is the coherence length of light for scattering by the rough part of the surface and $\Lambda$ is the wavelength of the periodic part of the surface. In our earlier works we have shown that by intensity measurements alone, the limit of detection can be taken to a much lower value of $\left(r_{0} / \Lambda\right)$, when the extended matched filtering method is employed. In this paper we follow the extended matched filtering method, and try to reach the lowest possible value of detection in $\left(r_{0} / \Lambda\right)$ by fitting the data to a polynomial. It is concluded by our numerical work that the lowest possible limit for detection from intensity measurements alone is $\left(r_{0} / \Lambda\right)=0.11$.
\end{abstract}

Keywords. Light scattering; matched filter; curve fit.

PACS Nos 42.25.Fx; 42.30.Kq; 42.30.Sy

\section{Introduction}

Light scattering and light intensity measurements have been one of the most useful and hence popular sources of getting information about a surface profile of material objects. As opposed to the more complicated techniques like interferometry, this technique allows ease of measurement and analysis. The accuracy of the results may not always match the more complicated methods but the results are obtained quickly and easily and hence this method serves as a very useful tool. As regards applications, among other things, light scattering has been used advantageously in various display devices and diffusers. It has also been used in certain medical imaging applications, for both of which it is important to know the nature of the surface profile. 


\section{$S$ Chatterjee and $V C$ Vani}

Detection of the periodic structure (called 'signal') in a surface, hidden behind roughness, becomes difficult and sometimes impossible if the randomness (called 'noise') is 'large'. This depends, in most cases, on the nature of the 'signal', described by the amplitude and the wavelength of the periodic part and also on the extent of 'noise', described by the coherence length of the radiation, obtained by the scattering of light by the rough part of the surface. Extracting the signal from noise uses various techniques and is an essentially interdisciplinary field making it a separate branch of study altogether.

In this paper we deal with a signal consisting of a single periodic structure. The random structure in this case is considered to be of Gaussian type, though the correlation function of the randomness can be any power law, as described below. This is obviously a special case for the randomness may have a more complex correlation function but will still be a useful practical description of the rough part. We intend to extend the results for more generalized and composite randomness. In this particular case of scattering of light from a periodic surface in the presence of randomness, earlier predictions had put the limit of detectability to be $\left(r_{0} / \Lambda\right) \sim$ 0.33 where $r_{0}$ is the coherence length of the rough part and $\Lambda$ is the wavelength of the periodic part. That is, if $\left(r_{0} / \Lambda\right)$ value is less than 0.33 , it becomes impossible to detect the signal $[1,2]$.

In our previous studies [3-8] we have shown that we can go far beyond the above limit of detectability if we use a different method of analyzing the data called the extended matched filtering method. The necessary details of this technique are explained in later sections. This method essentially involves the identification, first, of the zeroth-order peak, in the light scattering data and then eliminating it to determine the first-order peak, the underlying hypothesis being that the first-order peak, if it is submerged by the zeroth order one, must have the same shape as the far more intense zeroth order one. The purpose of this paper is to extend the established limit of detectability further, by fitting the data to a polynomial. In [8], we had stopped the detection exercise, when the scatter in the data became significant. Fitting the data to a polynomial and extending the range of data collection can in certain situations become a way of overcoming the limitation due to scattering of data, in the sense that it can help in defining the shape of the curve, guided by the earlier trend in the data. As will be seen in later sections, identification of minimum in $\Delta^{2}$ forms a very important part of our method of data analysis. We use the polynomial fit for the purpose, to ascertain more clearly the shape of the $\Delta^{2}$ vs. $c$ curve. We notice that for large values of the randomness this becomes an impossible exercise, and that determines the limit of detectability.

For the sake of completeness, we repeat the theory behind this analysis, briefly, in the next section ([3] gives more details). We also summarize our findings till now for two reasons. Firstly, we would like to give a complete picture of our work and secondly, this paper deals with the ultimate limit of detection achievable (using the present method of data analysis) when a periodic surface consisting of single periodicity is overlapped by randomness, for which, it is essential to know the previous results. The results of our data analysis and the conclusions drawn are presented in the sections following the theory. 


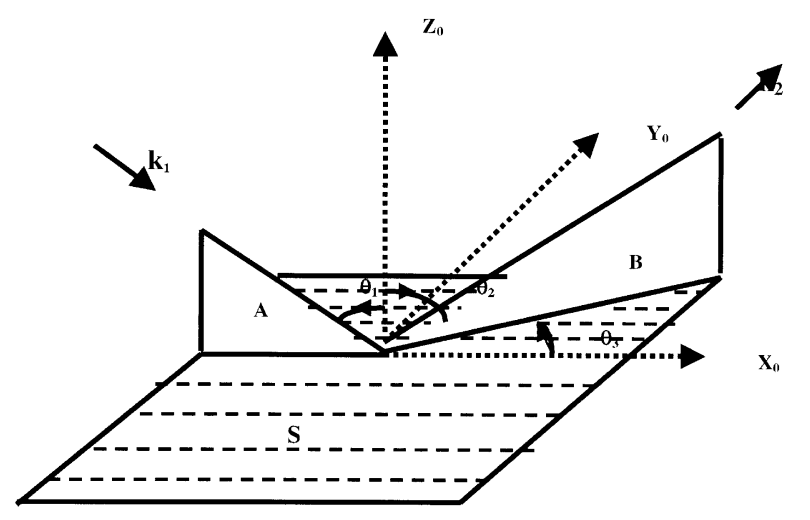

Figure 1. Geometry of scattering from the rough grating surface.

\section{Theory}

We consider a reflection grating, lying in the $x y$ plane, where elevations in the $z$-direction are given by,

$$
\xi(x, y)=a \cos (Q x)+\delta \xi(x, y) \ldots,
$$

where $Q=2 \pi / \Lambda$.

The random part is assumed to be a zero mean Gaussian stationary random process and has the correlation function

$$
\left\langle\delta \xi(x, y) \delta \xi\left(x^{\prime}, y^{\prime}\right)\right\rangle=\sigma^{2} \Phi(r),
$$

where

$$
r=\left[\left(x-x^{\prime}\right)^{2}+\left(y-y^{\prime}\right)^{2}\right]
$$

and $\Phi(r)$ is a monotonically decreasing function, with $\Phi(0)=1$. It is clear that the quantity $\sigma$ designates a measure of the height of the irregularities $\delta \xi(x, y)$. It is usual to find that beyond a distance $r>l$ the irregularity $\delta \xi(x, y)$ has no knowledge of that of $\delta \xi\left(x^{\prime}, y^{\prime}\right)$. Thus the correlation function $\Phi(r)$ falls rapidly for $r \gg l$. The quantity $l$ is referred to as the 'correlation length'. The coherence length $r_{0}$ of the reflected wave can be calculated from $\sigma$ and $l$, when the nature of the correlation function $\Phi(\mathbf{r})$ is known.

The scattering geometry is schematically described in figure 1 . The directions of the incident and scattered rays are expressed in the polar coordinates as $\left(\theta_{1}, 0\right)$ and $\left(\theta_{2}, \theta_{3}\right)$ as shown in figure 1 . Further, the wave vectors of scattering are defined as

$$
\begin{aligned}
& k_{x}=k\left(\sin \theta_{1}-\sin \theta_{2} \cos \theta_{3}\right), \quad k_{y}=-k \sin \theta_{2} \sin \theta_{3}, \\
& k_{z}=-k\left(\cos \theta_{1}+\cos \theta_{2}\right), \quad k_{x y}^{2}=k_{x}^{2}+k_{y}^{2},
\end{aligned}
$$

where $k=2 \pi / \lambda, \lambda$ being the wavelength of light. In the 'optics literature' the quantities $k_{x}, k_{y}$ and $k_{z}$ are often denoted by $v_{x}, v_{y}, v_{z}$ respectively, a convention that was followed in our earlier papers. 


\section{$S$ Chatterjee and $V C$ Vani}

In what follows, we shall calculate the scattered intensity under the Kirchoff approximation $[9,10]$. We consider the quantity $\left\langle\rho \rho^{*}\right\rangle_{0}$ as the intensity of light scattered by the rough surface in the direction $\left(\theta_{2}, \theta_{3}\right)$ divided by the intensity of light scattered by a smooth surface in the specular direction. On defining $\sqrt{ } g=$ $k_{x} \sigma / \sqrt{ } 2, \sqrt{ } g_{1}=a k_{z} / \sqrt{ } 2$, it is seen that

$$
\begin{aligned}
\left\langle\rho \rho^{*}\right\rangle_{0}= & \left\{J_{0}^{2}\left(\sqrt{ }\left(2 g_{1}\right)\right) f\left(k_{x}, k_{y} ; g\right)+\sum_{n=1}^{\infty} J_{n}^{2}\left(\sqrt{ }\left(2 g_{1}\right)\right)\left[f\left(k_{x}+n Q, k_{y} ; g\right)\right.\right. \\
& \left.\left.+f\left(k_{x}-n Q, k_{y} ; g\right)\right]\right\} B\left(\theta_{1}, \theta_{2}\right)
\end{aligned}
$$

where

$$
\begin{aligned}
& f\left(k_{x}, k_{y} ; g\right)=(2 \pi / A) \int \exp (-g[1-\Phi(r)]) J_{0}\left(k_{x y} r\right) r \mathrm{~d} r \\
& B\left(\theta_{1}, \theta_{2}\right)=\left[F_{3}\left(\theta_{1}, \theta_{2}, \theta_{3}\right)\right]^{2} S\left(\theta_{1}, \theta_{2}\right) \\
& F_{3}\left(\theta_{1} ; \theta_{2}, \theta_{3}\right)=\frac{\left(1+\cos \theta_{1} \cos \theta_{2}-\sin \theta_{1} \sin \theta_{2} \sin \theta_{3}\right)}{\left(\cos \theta_{1}\left(\cos \theta_{1}+\cos \theta_{2}\right)\right)} \\
& S\left(\theta_{1}, \theta_{2}\right)=S\left(\theta_{1}\right) S\left(\theta_{2}\right)
\end{aligned}
$$

with

$$
\begin{aligned}
& S(\theta)=\exp [(-1 / 4) \tan \theta \operatorname{erfc}(K \cot \theta)], \\
& K^{2}=(a Q)^{2}+4(\sigma / l)^{2} .
\end{aligned}
$$

The extended matched filter method described below, gives a method by which the best fit is made in order to extract the terms $J_{n}^{2}\left(\sqrt{ }\left(2 g_{1}\right)\right)$. The expressions given in (6) and (7) show that as the width $\left(1 / r_{0}\right)$ of $f\left(k_{x}, k_{y} ; g\right)$ increases, the zerothorder peak is broad enough to encompass other peaks too and the detection of the periodic part becomes impossible. It was noted by Baltes and others, that for a Gaussian correlation function, for the randomness, the periodic part is undetectable if $\left(r_{0} / \Lambda\right) \leq 0.33$ where $Q=2 \pi / \Lambda, \Lambda$ being the wavelength of the grating structure. With the help of the extended matched filter method we extract the amplitude $a$ and the wavelength $\Lambda$ (or the wave vector $Q$ ) of the periodic part, even in cases where $\left(r_{0} / \Lambda\right) \ll 0.33$, i.e. even in cases which do not permit identification of the periodic part by normal intensity measurements but only by intensity interferometry. We have found in earlier communications [3-8] that the values of $a$ and $\Lambda$ found with measurements at different wavelengths $\lambda$ come out to be consistently same, in the $n=1$ case. This shows the existence of the first lobe. The second lobe is far more difficult to find, since its intensity is of the order of $(a / \lambda)^{4}$ and is extremely low in the case under study.

Since the periodicity of the surface is confined only to the $x$-direction, we consider only the scattering along $k_{y}=0$ and from now on designate $f\left(k_{x}, 0 ; g\right)$ as $f\left(k_{x}\right)$.

The paper gives us a new method for resolution enhancement. It is based on the a priori hypothesis that a hidden periodicity, if it exists, is completely hidden by randomness. The nature of the profile $f\left(k_{x}\right)$ is however taken to be 
$f\left(k_{x}\right)=\left[1+(c / 2 x) k_{x}^{2}\right]^{x}$ as can be the case for widely different types of randomness, i.e., for widely different forms for the correlation function $f(r)$. The nature of the randomness is not known and hence the parameters $c$ and $x$ in the profile are unknown but are to be determined to match the case, which gives the best fit. Presence of side lobes of shapes similar to $f\left(k_{x} \pm Q\right)$ is the central theme in the extended matched filtering method described in the present paper.

We begin by considering that for $\sqrt{ } g_{1} \ll 1$, the amplitudes of the successive $n$th peaks fall as $\left(\sqrt{ } g_{1}\right)^{2 n}$ while the width of the peaks vary as $\Delta k_{x} \approx r_{0}^{-1}$. The separation of the peaks being $\delta k_{x}=Q$, the central $n=0$ peak can submerge all the higher order peaks for $\Delta k_{x} \gg \delta k_{x}=Q$. This method envisages the shape $f\left(k_{x}, 0 ; g\right)$ of the central peak and separates it out from the total intensity profile. This way it tries to identify the $n= \pm 1$ peaks, whose shape must match with that of the $n=0$ peak that has been eliminated out.

We begin by assuming (this is the shape of the central peak, which must be the same for all other peaks too)

$$
f\left(k_{x}\right)=f_{a}\left(k_{x}\right) \equiv\left[1+(b / 2 y) k_{x}^{2}\right]^{y} .
$$

We note that on defining

$$
\begin{aligned}
& Z\left(k_{x}\right)=\left[\left\langle\rho^{*}\left(k_{x}\right) \rho\left(k_{x}\right)\right\rangle /\left\langle\rho^{*}(0) \rho(0)\right\rangle\right]-f_{a}\left(k_{x}\right), \\
& \chi\left(k_{x}\right)=Z\left(k_{x}\right) / Z_{\max }
\end{aligned}
$$

we find,

$$
\chi\left(k_{x}\right)=\left(N_{0}+N_{1}+N_{2}\right) /\left(D_{0}+D_{1}+D_{2}\right)
$$

where

$$
\begin{aligned}
& N_{0}=J_{0}^{2}\left[f\left(k_{x}\right)-f_{a}\left(k_{x}\right)\right] \\
& N_{1}=J_{1}^{2}\left[f\left(k_{x}+Q\right)+f\left(k_{x}-Q\right)-2 f_{a}\left(k_{x}\right) f(Q)\right] \\
& N_{2}=J_{2}^{2}\left[f\left(k_{x}+2 Q\right)+f\left(k_{x}-2 Q\right)-2 f_{a}\left(k_{x}\right) f(2 Q)\right] \\
& D_{0}=J_{0}^{2}\left[f(Q)-f_{a}\left(Q^{*}\right)\right] \\
& D_{1}=J_{1}^{2}\left[f\left(Q^{*}+Q\right)+f\left(Q-Q^{*}\right)-2 f_{a}\left(Q^{*}\right) f(Q)\right] \\
& D_{2}=J_{2}^{2}\left[f\left(Q^{*}+2 Q\right)+f\left(Q^{*}-2 Q\right)-2 f_{a}\left(Q^{*}\right) f(2 Q)\right]
\end{aligned}
$$

and $Q^{*}$ is the wave vector at which $Z\left(k_{x}\right)$ is a maximum.

The existence of a hidden periodicity demands that the shape of the side lobe must exactly match that of the central peak. This 'matching' is sought through a best-fit condition, in which the estimator $\Delta^{2}$ estimates, in a 'standard deviation' sense, how far the hypothesis $\chi_{a}\left(k_{x}\right)$ really differs from the correct situation as given by $\chi\left(k_{x}\right)$. It is indeed correct that at $k_{x}=Q^{*}, \chi_{a D}\left(Q^{*}\right)=\chi_{a N}\left(Q^{*}\right)$ making $\chi_{a}\left(Q^{*}\right)=1$, identically, which is an important condition for the matched filtering that follows.

We find that close to the peak we can expand 


\section{$S$ Chatterjee and V C Vani}

$$
\begin{aligned}
f\left(k_{x}\right) \approx & 1-(c / 2) k_{x}^{2}+(1 / 2)\left[(c / 2 x)\left(k_{x}^{2}\right)\right]^{2} x(x-1) \\
& +1 / 6\left[(c / 2 x)\left(k_{x}^{2}\right)\right]^{3} x(x-1)(x-2) .
\end{aligned}
$$

This means that the radius of curvature of $f\left(k_{x}\right)$, close to the peak equals $c^{-1 / 2}$, while the exponent $x$ describes essentially the tail of the function. This means that close to the peak the shape of $f\left(k_{x}\right)$ is quadratic in $k_{x}$, while the dependence on $x$ starts for higher values of $x$. The coherence length $r_{0}$ beyond which $f\left(k_{x}\right)$ falls rapidly is given by $r_{0} \sim c / 2 x$.

It is clear that when our assumed $f_{a}\left(k_{x}\right)$ is very close to the actual $f\left(k_{x}\right)$, the terms $N_{0}$ and $D_{0}$ become negligible and the $N_{1}$ terms show prominent peaks at $k_{x}=$ $\pm Q \approx \pm Q^{*}$, and these lobes should match, in shape, with the chosen $f_{a}\left(k_{x} \pm Q^{*}\right)$. To quantitatively select the best match, we note that if the terms $N_{0}$ and $D_{0}$ be negligible (because of the match $f\left(k_{x}\right) \approx f_{a}\left(k_{x}\right)$ ) we must have $\chi\left(k_{x}\right)-\chi_{a}\left(k_{x}\right)$ to be extremely small, where $\chi_{a}\left(k_{x}\right)$ is defined as

$$
\chi_{a}\left(k_{x}\right)=\chi_{a N}\left(k_{x}\right) / \chi_{a D}\left(k_{x}\right)
$$

with

$$
\begin{aligned}
& \chi_{a N}\left(k_{x}\right)=\left[f_{a}\left(k_{x}+Q^{*}\right)+f_{a}\left(k_{x}-Q^{*}\right)-2 f_{a}\left(k_{x}\right) f_{a}\left(Q^{*}\right)\right], \\
& \chi_{a D}\left(k_{x}\right)=\left[f_{a}\left(Q^{*}+Q^{*}\right)+f_{a}\left(Q^{*}-Q^{*}\right)-2 f_{a}\left(Q^{*}\right) f_{a}\left(Q^{*}\right)\right] .
\end{aligned}
$$

The matched filtering is effected by defining an estimator

$$
\Delta^{2}=\int_{0}^{K}\left|\chi_{a}\left(k_{x}\right)-\chi\left(k_{x}\right)\right|^{2} \mathrm{~d} k_{x}
$$

Both $\chi_{a N}\left(k_{x}\right)$ and $\chi_{a D}\left(k_{x}\right)$ and hence $\chi_{a}\left(k_{x}\right)$ can be calculated from the above definitions while $\chi\left(k_{x}\right)$ is found from the data through $(15)$. We select the filter by choosing $b$ and $y$ to be the one which gives the minimum $\Delta^{2}(b, y)$. This is our criterion of the best fit for the matched filter in the least square sense. In all the results reported below, we have used $K=Q^{*}$. Also note must be taken of the fact that while $\chi\left(k_{x}\right)$ is a quantity which is found from the experimental data, $\chi_{a}\left(k_{x}\right)$ is a quantity that is given by our hypothesis, so that $\Delta^{2}$ gives a least square estimate of the deviation of the data from the hypothesis, under consideration. The main aim in the extended matched filtering method is to anticipate $f\left(k_{x}\right)$ first such that the shape of $f_{a}\left(k_{x}\right)$ describing the $f\left(k_{x}\right)$ must also match the shapes of the side lobes at $n= \pm 1$, where these side lobes are to be found by searching for the minimum of $\Delta^{2}(b, y)$.

Identification of the matched filter enables us to 'detect', through a least square fit, the parameters for the rough part of the surface. To find those for the periodic part, we use expression (10) to give (on defining $f^{\prime}\left(k_{x}\right)=\partial f / \partial k_{x}$ )

$$
\begin{aligned}
& f_{a}^{\prime}\left(Q^{*}+Q\right)+f_{a}^{\prime}\left(Q^{*}-Q\right)-2 f_{a}(Q) f_{a}^{\prime}(Q) \\
& =-\left(J_{2} / J_{1}\right)^{2}\left[f^{\prime}\left(Q^{*}+2 Q\right)+f^{\prime}\left(Q^{*}-2 Q\right)-2 f_{a}^{\prime}\left(Q^{*}\right) f(2 Q)\right] \\
& Z_{\max } J_{0}^{2}-\left[f_{a}(0)+f_{a}\left(2 Q^{*}\right)-2 f_{a}^{2}\left(Q^{*}\right)+2 f_{a}\left(Q^{*}\right) Z_{\max }\right] J_{1}^{2} \\
& \quad-\left[f_{a}\left(3 Q^{*}\right)+f_{a}\left(Q^{*}\right)-2 f_{a}\left(2 Q^{*}\right) f_{a}\left(Q^{*}\right)-2 f_{a}\left(Q^{*}\right) Z_{\max }\right] J_{2}^{2}=0
\end{aligned}
$$

where we have used the approximation, $f\left(k_{x}\right)=f_{a}\left(k_{x}\right)$. 
Detection of hidden periodicities by a matched filter
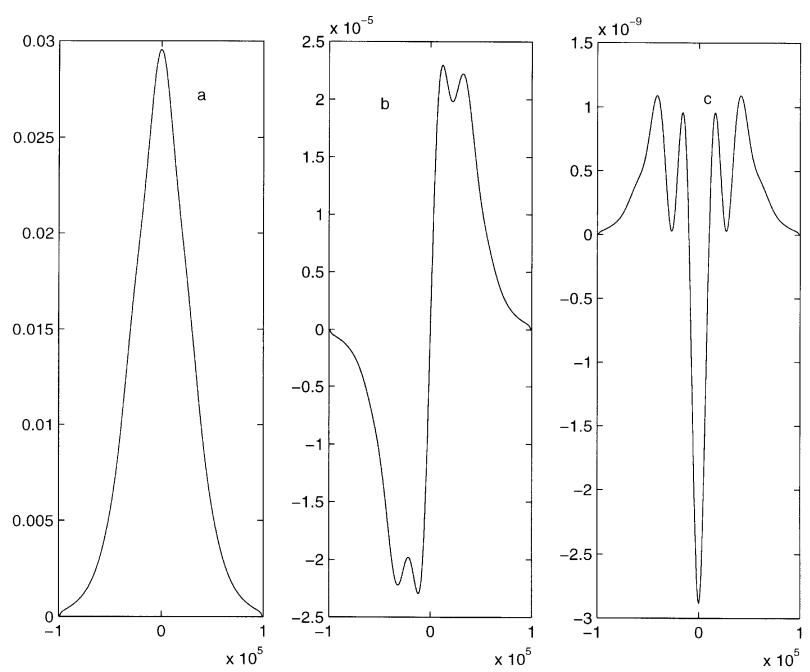

Figure 2. $\left\langle\rho \rho^{*}\right\rangle_{0}$ and its derivatives. In all three figures $k_{x}$ is on the $x$-axis. (a) $\left\langle\rho \rho^{*}\right\rangle_{0}$ (arbitrary units) vs. $k_{x}$, (b) first derivative vs. $k_{x}$ and (c) second derivative vs. $k_{x}$, for parameters in the text.

The parameters $Q^{*}$ and $\left(J_{1} / J_{0}\right)^{2}$ are solved by successive approximations by identifying $J_{2}^{2} \ll J_{1}^{2} \ll J_{0}^{2}$.

\section{Numerical results and discussion}

The results obtained earlier form the content of refs [3-8]. We now summarize these findings. We illustrate the algebraic procedure by 'simulating' numerically the problem of scattering by a rough grating, using the following choice of parameters: $\lambda=6328 \AA, a=328 \AA, \Lambda=6.25 \times 10^{-4} \mathrm{~cm}, Q=1.0057 \times 10^{4} \mathrm{~cm}^{-1}, x=3 / 2$, i.e. we consider a Cauchy-type correlation function for the disorder. As mentioned earlier in the introduction, we consider a single periodicity buried in a Gaussian noise.

The intensity of the scattered light in such a configuration is shown in figure 2a. It is clear that the intensity curve has only the central maximum and no other structure is visible. This can mean three things.

1. There is no extra structure present and the intensity curve has shown everything that needs to be observed.

2. Any periodicity, if it exists, is buried in this central maximum and the periodicity in the system is undetectable.

3. This situation also implies, based on the input from theory, that, any peak, if present and be subsumed by the central peak, must have the same shape as the central peak itself.

The derivatives of the intensity are shown in figures $2 \mathrm{~b}$ and $2 \mathrm{c}$. The derivatives show additional structures that are absent in the intensity curve in 2a. The existence of 


\section{$S$ Chatterjee and $V C$ Vani}
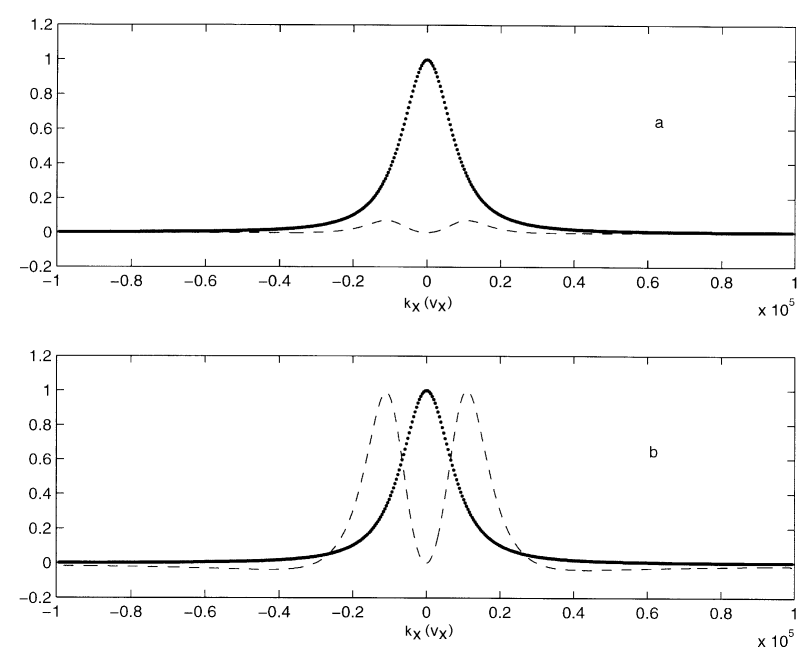

Figure 3. Comparison between $f_{a}\left(k_{x}\right)$ and $Z\left(k_{x}\right)$ and $\chi\left(k_{x}\right) . k_{x}\left(v_{x}\right)$ is the $x$-axis. (a) Dotted curve: $f_{a}\left(k_{x}\right)$ vs. $k_{x}$; broken curve: $Z\left(k_{x}\right)$ vs. $k_{x}$. (b) Dotted curve: $f_{a}\left(k_{x}\right)$ vs. $k_{x}$; broken curve: $\chi\left(k_{x}\right)$ vs. $k_{x}$.

these added structures shows clearly that the quantities $\left\langle\rho \rho^{*}\right\rangle_{1}$ and $\left\langle\rho \rho^{*}\right\rangle_{2}$ give rise to structures that are absent in $\left\langle\rho \rho^{*}\right\rangle$ as there is no additional input from outside. But this method may not be very satisfactory because the derivatives are known to add noise to the system and this happens because the derivative is taken not only of the signal but also of the noise already present.

This actually forms just the preliminary step of our analysis and we go on to the matched filter analysis from here.

Figure 3a shows $Z\left(k_{x}\right)$ plotted against $k_{x}$ and its comparison with $f_{a}\left(k_{x}\right)$ when plotted against $k_{x}$. Figure 3b shows $\chi\left(k_{x}\right)$ vs. $k_{x}$ and its comparison with $f_{a}\left(k_{x}\right)$ vs. $k_{x}$. These quantities are defined in the section on the theory. It is clear that $\chi\left(k_{x}\right)$ is a normalized version of $Z\left(k_{x}\right)$. These graphs provide the basic step in our matched filter analysis. It is seen that the peak in $\chi\left(k_{x}\right)$ is shifted from the origin and the position of the peak gives us $Q^{*}$. As $Q^{*}$ defines the position of the first peak, $2 Q^{*}$ should give the position of the second peak and $3 Q^{*}$ and $4 Q^{*}$ correspond to the third and fourth peaks and so on. We know, $Q^{*}=(2 \pi / \Lambda)$ where $\Lambda$ is the wavelength of the periodic part of the surface. Having known the position, for instance, of the first peak, one has to determine the other parameters, namely $a$, required for the correct identification of the periodic structure. One thus defines $\Delta^{2}$, as in the section on theory. The choice of the best fit is ascertained by checking $\Delta^{2}$, as defined in (22). The point where $\Delta^{2}$ has a minimum corresponds to the case where the hypothesis has the minimum deviation from the data. The position where $\Delta^{2}$ is a minimum gives us all the required parameters to enable correct identification.

The improved detectability is related to the central point in matched filtering that the $f_{a}\left(k_{x}\right)$ selected must give a satisfactory match to $f\left(k_{x}\right)$. From the parameters of randomness used in our numerical simulations the quantity $r_{0}^{2}$ is calculable and is expected to follow $\left(\lambda^{2} r_{0}\right)=$ constant. Correct identification of matched filter would 
Detection of hidden periodicities by a matched filter

Table 1. Parameters at different wavelengths.

\begin{tabular}{lccccc}
\hline $\begin{array}{l}\text { Wavelength } \\
\lambda(\AA)\end{array}$ & $Q\left(10^{4} \mathrm{~cm}^{-1}\right)$ & $\begin{array}{c}a \text { (calculated) } \\
(\AA)\end{array}$ & $r_{0} / \Lambda$ & $\begin{array}{c}\text { Error in } Q \\
(\%)\end{array}$ & $\begin{array}{c}\text { Error in } a \\
(\%)\end{array}$ \\
\hline 10000 & 0.994 & 520 & 0.4044 & -1.09 & 0.37 \\
7500 & 0.995 & 405 & 0.2275 & -0.99 & 4.62 \\
6943 & 1.02 & 343 & 0.1949 & 1.6 & 1.3 \\
6328 & 1.05 & 304 & 0.1619 & 4.5 & 7 \\
6000 & 1.001 & 288 & 0.1456 & -0.40 & 7.7 \\
5700 & 0.99 & 304 & 0.1314 & -1.5 & 10 \\
5000 & 1.040 & 200 & 0.1011 & 3.48 & 24.7 \\
\hline
\end{tabular}

Table 2. Parameters at different $r_{0} / \Lambda$ for wavelength $6328 \AA$.

\begin{tabular}{|c|c|c|c|c|c|}
\hline \multicolumn{2}{|c|}{$b$ and $y$ at $\Delta_{\min }^{2}$} & \multirow{2}{*}{$\begin{array}{c}r_{0} / \Lambda \\
\text { (actual) }\end{array}$} & \multirow{2}{*}{$\begin{array}{c}r_{0} / \Lambda \\
\text { (calc.) }\end{array}$} & \multirow{2}{*}{$\begin{array}{c}a \text { (calc.) } \\
\left(* 10^{-8}\right)\end{array}$} & \multirow{2}{*}{$\begin{array}{c}\text { Error } \\
\text { in } a(\%)\end{array}$} \\
\hline$b$ & $y$ & & & & \\
\hline $26.0 * 10^{-8}$ & 1.5 & 0.4858 & 0.4710 & 326 & 0.51 \\
\hline $17.0 * 10^{-8}$ & 1.5 & 0.3886 & 0.3808 & 325.8 & 0.61 \\
\hline $12.0 * 10^{-8}$ & 1.6 & 0.3239 & 0.3100 & 326 & 0.51 \\
\hline $8.0 * 10^{-8}$ & 1.5 & 0.2591 & 0.2612 & 333 & 1.63 \\
\hline $4.3 * 10^{-8}$ & 1.5 & 0.1943 & 0.1915 & 320 & 2.35 \\
\hline $2.9 * 10^{-8}$ & 1.4 & 0.1619 & 0.1630 & 305 & 7.01 \\
\hline $2.1 * 10^{-8}$ & 1.4 & 0.1500 & 0.1386 & 277 & 18 \\
\hline $2.1 * 10^{-8}$ & 1.4 & 0.1401 & 0.1386 & 266 & 23 \\
\hline $1.8 * 10^{-8}$ & 1.5 & 0.1300 & 0.1239 & 267 & 23 \\
\hline $1.5 * 10^{-8}$ & 1.4 & 0.1205 & 0.1171 & 226 & 31 \\
\hline $1.3 * 10^{-8}$ & 1.4 & 0.1134 & 0.1089 & 216 & 34 \\
\hline $1.0 * 10^{-8}$ & 1.8 & 0.1004 & 0.0847 & 206 & 37 \\
\hline- & - & 0.0907 & - & - & - \\
\hline - & - & 0.0810 & - & - & - \\
\hline
\end{tabular}

demand that $\left(b / 2 y r_{0}^{2}\right)=1$ in every case. As $\left(r_{0} / \Lambda\right)$ reduces, the errors in finding $b$ and $y$ do contribute to large errors in finding $Q^{*}$ and $Z_{\max }$, due to smallness of $(a / \lambda)$ and hence the detectability suffers immensely. In our matched filtering, once the $n=0$ peak is filtered out we are to distinguish the two comparable peaks at $n= \pm 1$, but separated by a larger $\Delta k_{x}=2 Q^{*}$. The latter, according to the Rayleigh criterion or the Sparrow criterion, allows distinguishability of the peaks for much lower values of $r_{0}$.

Table 1 gives parameters for different wavelengths supporting the matched filter method. Next, to find the detection limit, $r_{0} / \Lambda$ is varied in each case by varying the correlation length of the rough surface. Table 2 , on the other hand, gives the details of the fit for a fixed wavelength of $6328 \AA$. The values of $r_{0} / \Lambda$ in different cases are varied by varying the correlation length. Tables 1 and 2 complement each other. The interest here is in observing the situation for lower values of $\left(r_{0} / \Lambda\right)$. 
This paper is part of our continued effort to reach lower values of detectability in $\left(r_{0} / \Lambda\right)$. Here, we fit the data to a polynomial to find a minimum. A brief discussion on curve fitting is necessary here. The best choice would have been to let the parameters float (to be determined by the fit) and choose a non-linear
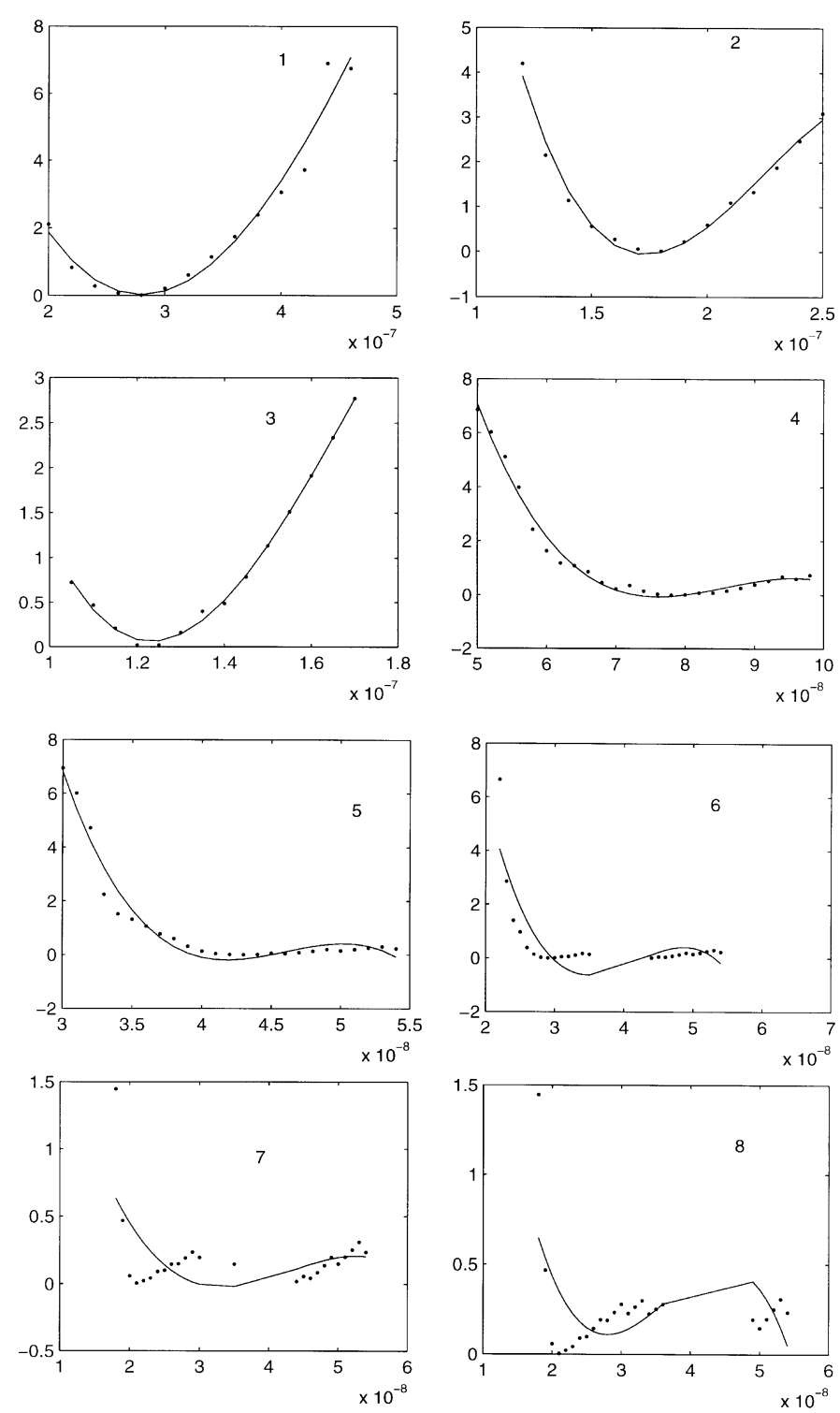

Figure 4. Twelve plots showing different detection limits. These are obtained by plotting $b$ ( $x$-axis) vs. $\Delta^{2}$ ( $y$-axis) for different $y$ values. $1-0.4858$, $2-0.3886,3-0.32,4-0.25,5-0.19,6-0.16,7-0.15,8-0,13,9-0.12$, $10-0.10,11-0.09,12-0.08$. 

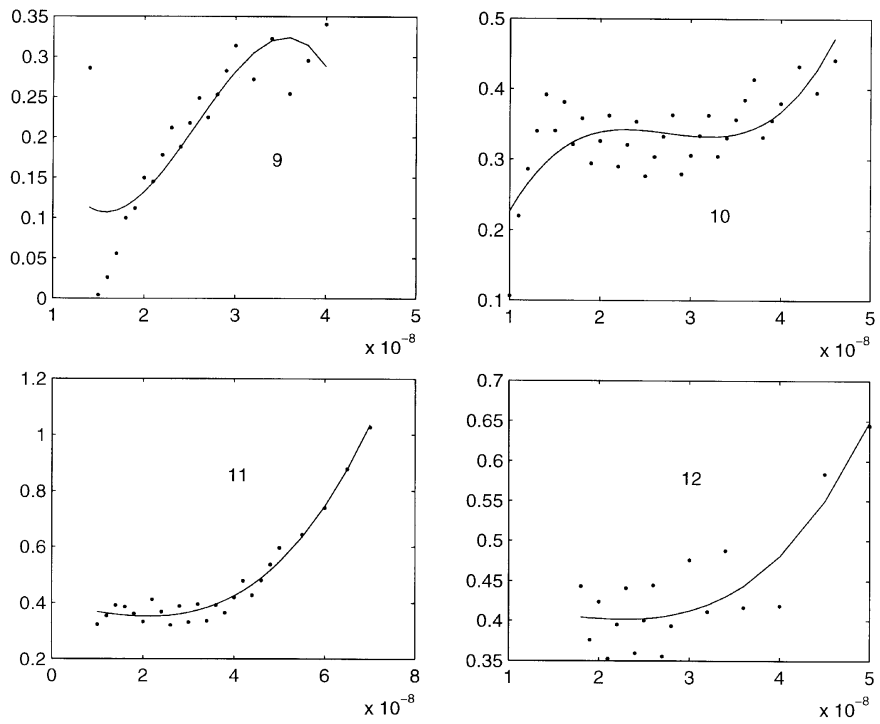

Figure 4. Continued.

data fitting. But this procedure can lead to erroneous results unless there are enough constraints on the parameters. Also, here the answer need not be unique. The existence of closely related physical minima (as opposed to the mathematical minimum) can lead to confusing results. A polynomial fit, on the other hand, is linear in the parameters and the answer is unique [11]. We have therefore chosen a polynomial to represent the trend in the data.

The simulated data are fitted to a polynomial of the following kind:

$$
Y=a(0)+a(1)^{*} x+a(2)^{*} x^{2}+a(3)^{*} x^{3},
$$

where $a(0), a(1), a(2), a(3)$ are the parameters to be determined, $Y$ represents $\Delta^{2}$ and $x$ denotes $b$. The polynomial fit gives a reasonably good representation of the data. That the fit is good is shown by the value of the reduced chi-square,

$$
\begin{aligned}
\text { Reduced chi-square }=\sum & \left(\left(1 /(\text { number of points })^{2}\right)\right. \\
& \left.(\text { calculated value }- \text { simulated value })^{2}\right)
\end{aligned}
$$

which is nearly unity. As one can easily see, the resolution in the simulated data has been reduced for $r_{0} / \Lambda<0.15$ and also the range in $b$ where data is generated has to be increased in order to find a clearly defined curve in the $b$ vs. '(delta)^ 2 ' (meaning $\Delta^{2}$ ) graph. This result is borne out by the fact that the errors become quite high as one reaches lower $\left(r_{0} / \Lambda\right)$ values. But the point in carrying out this exercise is to make the periodic structure detectable.

Up to $\left(r_{0} / \Lambda\right)=0.11$ we find a minimum in the $b$ vs. $\Delta^{2}$ curve. For values of $\left(r_{0} / \Lambda\right)=0.15$ and lower, we find a lot of scatter in the data. However, the existence of the minimum is clear until it reaches values close to 0.11 .

Figure 4 summarizes the results of our analysis. These figures together consist of 12 graphs showing the polynomial fit. In each case we have fitted the data to a 


\section{$S$ Chatterjee and $V C$ Vani}

polynomial of order three. Lower power terms alone were not sufficient to fit the data and higher power terms were found unnecessary. From the fit we find that as $\left(r_{0} / \Lambda\right)$ becomes less, (1) the numerical value of the minimum increases and (2) for $\left(r_{0} / \Lambda\right)<0.11$, the minimum is not clear any more.

For lower values of $\left(r_{0} / \Lambda\right)$ we find only a monotonic increase, but no clearly defined minimum. We therefore conclude that $\left(r_{0} / \Lambda \sim 0.11\right)$ forms the limit of detectability when the extended matched filtering technique is used.

\section{Conclusion}

It is to be noted that this problem is in some sense one of resolution enhancement and we have dealt with only one periodicity. In our opinion we have reached the limit of detection in the problem containing a single periodicity. As we go to higher order peaks (2nd, 3rd and so on), the intensity of the peaks drops very sharply and detection becomes very difficult. We will stop working on the single periodic structure at this stage. We have demonstrated quite convincingly that the range of detection in $\left(r_{0} / \Lambda \cong 0.11\right)$ happens to be much lower than the value predicted earlier. Multiple periodic structures occur more frequently in nature and naturally form a logical extension of this work. We anticipate that the detectability should deteriorate as the number of periodic structures buried in randomness go up. It should be interesting to see whether this method can resolve two closely placed peaks in a periodic structure, hidden behind randomness.

\section{References}

[1] J C Dainty and D Newman, Opt. Lett. 8, 608 (1983)

[2] H P Baltes, H A Ferwerda, A S Glass and B Steinle, Opt. Acta 28, 11 (1981)

[3] S Chatterjee, Indian J. Phys. B74, 363 (2000)

[4] S Chatterjee and V C Vani, Bull. Astr. Soc. India 30, 835 (2002)

[5] S Chatterjee and V C Vani, J. Mod. Opt. 50, 833 (2003)

[6] V C Vani and S Chatterjee, Bull. Astr. Soc. India 31, 457 (2003)

[7] V C Vani and S Chatterjee, Curr. Sci. 86, 177 (2004)

[8] V C Vani and S Chatterjee, Appl. Opt. 46, 3664 (2004)

[9] P Beckmann and A Spizzichino, The scattering of electromagnetic waves from random surfaces (Pergamon Press, London, 1963)

[10] P Beckmann, Scattering of light by rough surfaces, in Progress in optics edited by E Wolf (North Holland, 1967) Vol. 6, pp. 55-69

[11] P R Bevington Data reduction and error analysis for the physical sciences (McGraw Hill, New York, 1969) 